\title{
Proactive Behavior of an Autonomous Mobile Robot for Human-Assisted Learning
}

\author{
A. Garrell, M. Villamizar, F. Moreno-Noguer and A. Sanfeliu \\ Institut de Robòtica i Informàtica Industrial, CSIC-UPC \\ \{agarrell,mvillami,fmoreno,sanfeliu\}@iri.upc.edu
}

\begin{abstract}
During the last decade, there has been a growing interest in making autonomous social robots able to interact with people. However, there are still many open issues regarding the social capabilities that robots should have in order to perform these interactions more naturally. In this paper we present the results of several experiments conducted at the Barcelona Robot Lab in the campus of the "Universitat Politècnica de Catalunya" in which we have analyzed different important aspects of the interaction between a mobile robot and nontrained human volunteers. First, we have proposed different robot behaviors to approach a person and create an engagement with him/her. In order to perform this task we have provided the robot with several perception and action capabilities, such as that of detecting people, planning an approach and verbally communicating its intention to initiate a conversation. Once the initial engagement has been created, we have developed further communication skills in order to let people assist the robot and improve its face recognition system. After this assisted and online learning stage, the robot becomes able to detect people under severe changing conditions, which, in turn enhances the number and the manner that subsequent human-robot interactions are performed.
\end{abstract}

\section{INTRODUCTION}

Human-robot interaction (HRI) is an active research topic which has received much attention, both from academic and private communities. There is a wide range of applications in which HRI plays a fundamental role, from developing automatic exploration sites [18], to using robot formations to accompany people $[5,6,7]$.

Recent efforts are focused on creating robots able to start conversations with humans in a friendly and natural manner [12]. In this work, we go a step further, and after initiating a conversation, and therefore, creating an engagement with a person, we have provided a mobile robot with the capabilities to seek assistance from the person. In particular, we will show how the robot and person perform a collaborative task in which the robot asks the human to teach it to improve its face recognition system.

The contributions of the paper are therefore twofold. Firstly, we present a framework where a mobile robot is able to initiate a dialogue with a person and create an engagement. In this study, we focus on the way the robot initiates the communication with the person, which should be the most

Work supported by the Spanish Ministry of Science and Innovation under projects RobTaskCoop(DPI2010-17112) and the EU ARCAS Project FP7ICT-2011-287617

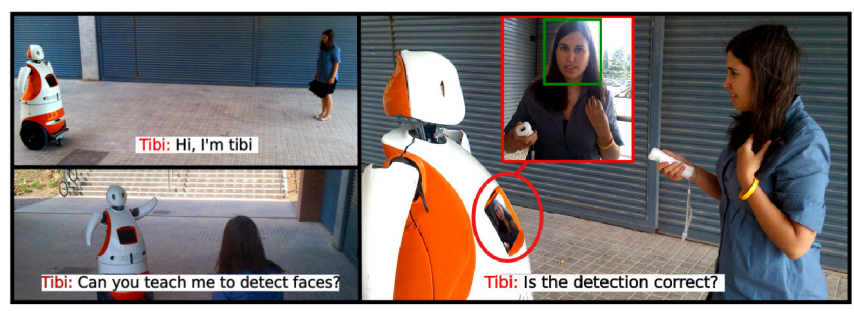

Fig. 1. Human-Robot Interaction and Communication. Left: Tibi robot approaches a person to initiate a conversation. Right: After the first contact, the person assists Tibi to improve its visual skills. A Wii's remote controller is used to help to validate and improve the visual face detector.

natural possible for the human. In particular, we consider the human communication model proposed by Clark [2], based on the notion that people in a conversation share the view for each other persons, such as a speaker, hearer, and side participants. In order to perform this initial task, we have provided the robot with a simple visual module, able to detect human faces in real-time, although under the requirement that faces have to be in a non-occluded and frontal position.

Our second contribution is that, once the engagement has been created, we propose a second robot-human communication framework, in which the human can naturally help the robot to improve the performance of the face recognition module. More specifically, we propose an online learning algorithm, assisted by the human, which increases the performance and robustness of the initial face detector, allowing to detect faces under harsh conditions, such as abrupt light change or partial occlusions. In addition, the robot learns the person's identity, with the aim of establishing future and coherent dialogues. In this online and real-time assisted learning algorithm, the human plays the role of a teacher, and guides the robot during its learning process, validating and correcting the output of the face recognizer. The amount of human intervention drops in time, and usually after a few seconds the robot visual system becomes very robust and reliable. Fig. 1 shows a typical scenario, with three different frames of this teaching process between a person and our robot Tibi.

The robot's capability to approach people and to learn using human assistance enables a number of applications. We believe that one promising application is the capacity of the robot to autonomously look for people who can assist it, and progressively improve its skills during the 
interaction process. In addition, this learning stage can be performed by any non-expert person as it only requires a simple communication with the robot to validate a few face candidates proposed by the face recognizer module.

The remainder of the paper is organized as follows. In section II, we present the related work. The tasks and behavior of the robot are specified in section III. In Section IV we describe the experimental setting and the evaluation methodology, which are subsequently used in the results section V. Finally, discussion and conclusions are given in sections VI and VII, respectively.

\section{RELATED WORK}

We next describe related work and split it according the two main contributions of our work: the methods that propose a natural and friendly human-robot engagement and the visual recognition algorithms that can be progressively adapted using both the observation of the incoming data and the corrections of a human teacher.

Natural Engagement. One important topic within the human-robot interaction research is that of providing the robots with the ability to begin an interaction with a human. Typically, it is assumed that social robots may engage in the same way as people do, using human-like body properties and gestures [14]. Recent studies present robots which are able to encourage people to begin interaction [4], while expecting people to approach them to initiate a dialogue.

On the other hand, there has also been research in developing robots able to start themselves the interaction with a human. Satake et al. [16] proposed a model of approaching behavior to initiate a conversation with walking pedestrians. Another topic that has been studied, is that of when is the appropriate moment to start the interaction or the participation [17]. This should be the situation before or just at the moment that both human and robot establish the common belief that they are sharing a conversation.

The spatial formation of people around a robot has also been taken into account to pick a person and initiate interaction. Several works consider the kind of motion of the people surrounding the robot, and decide to initiate conversation with a specific person depending on his/her trajectory or on the distance to the robot [11].

The social rules regarding the beginning of a conversation have been studied in several works. In [8], for example, it is suggested that these social rules are a ritual that mutually confirm the start of a conversation. Other works have addressed the greeting process. The comfortable direction of a robot approaching a person and the distance between speakers were studied in [21].

In this work, we intend to go a step further from previous approaches, and propose the robot to proactively seek for the interaction with a human, with the purpose of convincing the human to help it to improve its visual detections. The main issue to handle in this context is that the approached person might not understand that the robot is trying to initiate a conversation with him/her. Humans initiate their conversation by eye gaze [8], but in a real environment it is very difficult

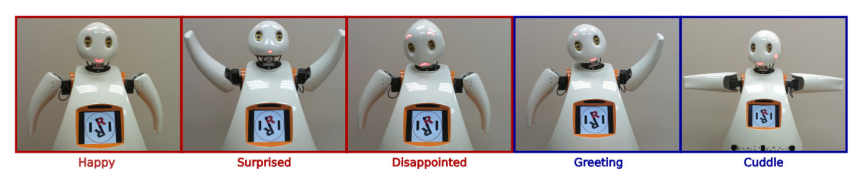

Fig. 2. Tibi Gestures. Movements performed by Tibi during experiments. Left: Three different emotional expressions. Right: Two actions.

for a robot to recognize human gazes. Instead, we use the body orientation, gestures and verbal interaction. Once, the human has understood the robot intentions, we have developed a simple and efficient communication protocol that will let the person teach the robot.

Human-Assisted Recognition. Object recognition is a hot topic in the computer vision community, with impressive results in detecting objects in challenging images and situations [19]. However, most of the methods are trained offline, either because they use large amounts of training data or because they require complex and time-consuming learning algorithms. In contrast, there are situations in which offline learning is not feasible, for instance when the training data is obtained continuously, or when the size of the training set is very cumbersome, making impractical a batch processing. These situations have been addressed by online learning methods that use their own predictions to train and update a classifier [13]. Yet, although these approaches have shown great adaptation capabilities, they are prone to suffer from drifting when updating the classifier with wrong predictions.

In order to make online learning algorithms more robust, recent approaches have proposed using the human assistance during the learning stage. In [20], a face classifier is computed on-the-fly, and progressively updated and improved using its own predictions and human corrections.

In this paper, we integrate the algorithm proposed in [20] within a robot platform, and provide the robot with the ability to learn from a human, using a communication process that requires almost no-human effort. We believe that the integration of this kind of high level learning algorithms in an autonomous mobile robot, and the development of the engagement strategies, are an important contribution for the HRI community.

\section{ROBOT's BEHAVIOR}

\section{A. Robot's Proactively Seeking Interaction}

Recent studies have focused on developing robots able to encourage people to begin interaction [4]. The most common strategy for robots is to expect people to approach them to initiate a dialogue. In contrast, as shown in Fig. 1, the ongoing research presents a robot that approaches people in a safe and friendly manner to begin a communication.

This strategy for creating people-to-robot engagements is more proactive than just waiting for the person to begin the interaction. In addition, the robot's ability to approach people opens up a wide range of applications. For instance, one might think about an invitation service, where a robot approaches people to offer city information and invite them to take a tour. Another possible application is what we propose in this paper, where we have exploited this proactive 
TABLE I

\begin{tabular}{|c|c|}
\hline & Assistance Expressions \\
\hline \multirow{2}{*}{$\begin{array}{l}\text { Invitation to } \\
\text { create and } \\
\text { engagement }\end{array}$} & $\begin{array}{l}\text { Hey, how are you? I am Tibi. I'm trying } \\
\text { to learn to detect faces, will you help me? }\end{array}$ \\
\hline & $\begin{array}{l}\text { Hi, I am Tibi, I'd like to learn how to } \\
\text { recognize different objects, can you be my teacher? }\end{array}$ \\
\hline \multirow{2}{*}{$\begin{array}{l}\text { Invitation to } \\
\text { continue the } \\
\text { interaction }\end{array}$} & Please, don't go. It will take just two minutes. \\
\hline & $\begin{array}{l}\text { Let me explain you the purpose of the experiment, } \\
\text { and then, you can decide if you want to stay. }\end{array}$ \\
\hline
\end{tabular}

Phrases Uttered by Tibi. Sample robot phrases to start interaction with a person.

behavior to improve the perception capabilities of the robot by enabling the human to teach it.

In order to let the robot by itself to begin the interaction with humans, we have used a laser range scanner to detect people in the space [1]. After this initial localization, the robot approaches the person keeping the distances of people's personal space. The robot is also able to respond according to human reactions. For example, if after the initial approach the robot invites the chosen person to come closer, and he/she ignores it, the robot will repeat the invitation. However, if the human does not come closer, the robot will search for another volunteer. If the person shows interest in the robot, it will start the interaction process.

The use of space we consider is based on the conceptual framework known as "proxemics", proposed by Hall [9]. This work considers the following taxonomy of distances between persons within a group of people:

- Intimate distance: the presence of another person is unmistakable, close friends or lovers $(0-45 \mathrm{~cm})$.

- Personal distance: comfortable spacing, friends $(45 \mathrm{~cm}-$ $1.22 \mathrm{~m})$.

- Social distance: limited involvement, non-friends interaction $(1.22 \mathrm{~m}-3 \mathrm{~m})$.

- Public distance: outside circle of involvement, public speaking $(>3 \mathrm{~m})$.

Based on these proxemics, Michalowski et al. [11] classified the space around a robot to distinguish human's levels of engagement while interacting or moving around a robot.In the present work, using phrases such as those of Table I, our robot tries to maintain the social distance as a first approach, and when the person has accepted the invitation to interact, he/she can move into the personal distance circle.

One of the main purposes of this work is to study which should be the robot's behavior to initiate an interaction with a human. Inspired in the literature of empathy and prosocial behavior [3] we have analyzed three different conducts: (1) The robot only performs verbal cues to communicate with the participants; (2) The robot performs verbal cues and nonverbal cues (arms' gestures and gaze); and (3) The robot performs verbal and nonverbal cues, and approaches humans.

Once the initial interaction is performed and the human has accepted, the goal is then to approach him/her, from a public distance level until a personal distance level. In order to encourage the person to move even closer, the robot performs the following actions depending on the aforementioned behaviors:

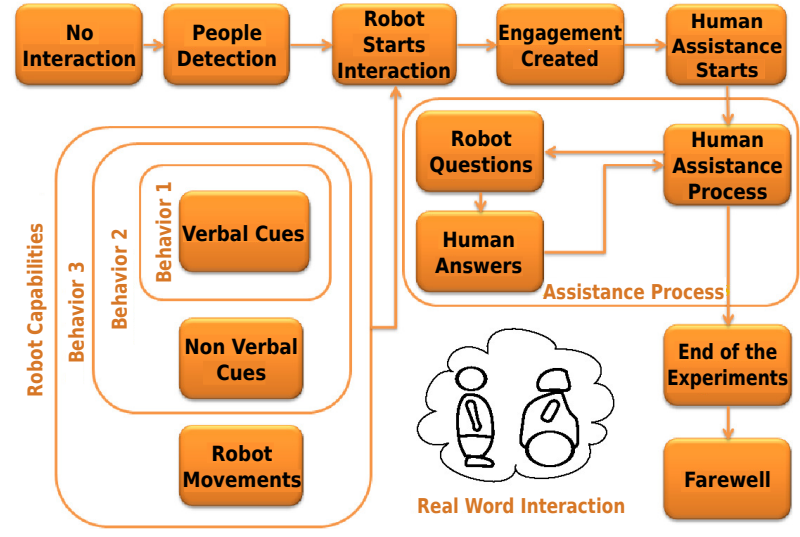

Fig. 3. Approach Overview. Sketch of the experiments performed to analyze different robot behaviors.

TABLE II

\begin{tabular}{|l|l|}
\hline \multicolumn{2}{|c|}{ Assistance Expressions } \\
\hline Assistance & $\begin{array}{l}\text { Is your face inside the rectangle? } \\
\text { I'm not sure if I see you, am I? }\end{array}$ \\
\hline No detection & $\begin{array}{l}\text { I can't see you, move a little bit. } \\
\text { Can you stand in front of me? }\end{array}$ \\
\hline Farewell & $\begin{array}{l}\text { Thank you for your help, nice to meet you. } \\
\text { I hope to see you soon. }\end{array}$ \\
\hline
\end{tabular}

Assistance Expression. Sample phrases uttered by the robot when it is updating the visual face classifier.

- Verbal communication: Encouragement comments, such as "Don't be afraid I just want to talk to you", "Can you teach me to detect faces?"

- Non-verbal communication: Gestures, arms and neck movements. A few samples are shown in Fig. 2.

- Robot motions: The robot approaches the person until reaching a social distance level.

Each one of these strategies might have a different impact in different users. For that reason, a set of experiments has been performed to analyze the acceptability of each behavior. A diagram of the different strategies is depicted in Fig. 3.

\section{B. Online Human-Assisted Face Recognition}

Once the robot has created the engagement with a human, we propose an approach in which the classifier used to initially detect the face is progressively updated and enhanced using the human assistance. The amount of human intervention is minimized and integrated within the online learning procedure, such that in a few seconds, complex face appearances can be learned.

In order to perform the human assistance, we equipped our robot with a screen that depicts the results of the online classifier, see Fig. 1. When one of the frames in the input video contains a face candidate for which the classifier is not confident, the robot asks for the human assistance through a set of precise and non-technical questions, that just expect a 'yes' or 'not' answer using a Wii remote control. Table II shows examples of such questions. This Wii remote control is introduced by the robot, Tibi is able to explain that a person in charge of the experiments will give to the participants the Wii remote control and how it must be used. 


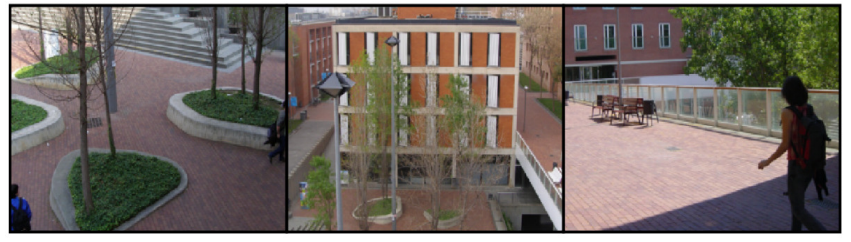

Fig. 4. Urban Environment. Some views of the scenario used in our experiments at the Barcelona Robot Lab.

\section{Method}

With the purpose of providing a convincing validation, we have performed our analysis on real scenarios. We chose the Barcelona Robot Lab. The conducted robot's tasks were: (1) approach a person to start the interaction and increase interest in helping the robot; and (2) invite the person to help and enhance the robot's face recognition system.

\section{A. Scenario}

The urban area considered for the tests is the Barcelona Robot Lab in the campus of the Universitat Politècnica de Catalunya (UPC), with an approximate size of $10.000 \mathrm{~m}^{2}$. Fig. 4 shows a few snapshots of this area. During the experiments, the robot was randomly navigating across this area while looking for people to initiate the interaction.

\section{B. Robot}

For the experiments, we used a mobile service robot, Tibi, specially designed to operate in urban pedestrian areas. It was originally developed in the European URUS (Ubiquitous Networking Robotics for Urban Sites) project [15]. Tibi, shown in Fig. 2 and Fig. 2, is based on the two-wheeled, selfbalancing Segway RMP200 platforms. The Segway RMP200 is, in many ways, an ideal platform to run in urban areas. Note that Humanoid robots are not yet capable of operate in outdoor environments.

The Tibi robot is $165 \mathrm{~cm}$ tall, occupies a clearance space of $80 \mathrm{~cm}$, and weights $110 \mathrm{~kg}$. It is equipped with multiple sensors, including a Bumblebee stereo camera and three lasers. In order to initially detect the persons at large ranges we use the front laser mounted at $40 \mathrm{~cm}$ above the ground. This just yields a rough estimation of the person's pose. The precise localization of his/her face is performed with one of the stereo cameras. In addition, a touch screen is located at the front of the robot, it is used to communicate with people.

\section{Experimental Design}

In order to test our framework, we conducted the following three experiments, all of them with the Tibi robot moving around the University Campus:

- People's personal space: The first set of experiments aimed to obtain the persons' personal space preferences when they were staying at a certain point and were approached by the robot.

- Robot's behaviors: In the second experiment, we compared the different robot behaviors described in Sec. IIIA to initiate the interaction. Firstly, the robot only used voice instructions to attract people's attention. Secondly, the robot was allowed to turn around to look at people's
TABLE III

\begin{tabular}{|l|}
\hline \multicolumn{1}{|c|}{ Survey's Questions } \\
\hline General Robot Behavior Scale \\
How comfortable did you feel near the robot? \\
How safe did you feel around the robot? \\
\hline Robot's Sociability Scale \\
How social was the robot's behavior? \\
How natural was the robot's behavior? \\
\hline Robot's Intelligence Scale \\
How intelligent did the robot behave? \\
How well could the robot anticipate to your movements? \\
\hline
\end{tabular}

Piece of the Questionnaire. Some survey questions asked of each participant after each robot behavior.

position. And, finally, the robot had the capability to move towards the people to interact with them.

- Human-assisted face recognition: We analyzed the effect of the human assistance over the face recognition performance, and over the duration and comfortability of the human-robot interaction.

\section{Participants}

For the first experiment, we considered 15 volunteers between 20 and $40(\mathrm{M}=27.4, \mathrm{SD}=5.78)$ years old. None of them had experience working or interacting with robots.

For the other two experiments, we picked 30 people (16 women, 14 men) on the University Campus. Participants were ranging in age from 20 to 65 years $(\mathrm{M}=39.24$, $\mathrm{SD}=$ 12.86), and represented a variety of university majors and occupations including computer science, mathematics, biology, finance and chemistry. For each person, we randomly activated one of the three robot behaviors to start the interaction. Then, each participant assisted the robot to improve its visual skills (third experiment). Again, none of the participants had previous experience working or interacting with robots.

\section{E. Measures and Analysis}

People's personal space. We conducted a series of tests in which the robot moved towards a person at different speeds and angles. When the person felt that the was robot too close, he/she started walking away. At the moment the person moved away we recorded the robot-to-person distance using the frontal laser of the robot.

Robot's behaviors. Our independent variables considered whether the robot approached the person or if it only used voice instructions. The main dependent variables involved participants' perceptions of the robot's persuasiveness, their compliance with the robot's suggestions, and their perceptions of the robot's social and intellectual characteristics. Each of these fields, was evaluated by every participant using a questionnaire to fill out after the experiment, based on [10]. Some questions are presented in Table III. The measurement was a rating on a Linkert-scale between 1 to 7, from "Not at all" to "Very much". For the evaluation score, an analysis of variance (ANOVA) measurements was conducted.

The information given to the volunteers about the robot was minimal to start the experiment, and hence, their behavior was not predefined at all. Participants were told to behave naturally, listen to robot's instructions and help it. Volunteers could decide whether to stay and perform the experiment or 
TABLE IV

\begin{tabular}{|c|ccc|}
\hline \multicolumn{4}{|c|}{ People's Personal Space } \\
\hline \multirow{2}{*}{} & \multicolumn{3}{|c|}{ Tibi's velocity of approach } \\
\cline { 2 - 5 } & vel.=0.3 m/s & vel. $=0.6 \mathrm{~m} / \mathrm{s}$ & vel. $=0.9 \mathrm{~m} / \mathrm{s}$ \\
\hline$\alpha=45(\mathrm{deg})$ & $0.30( \pm 0.20)$ & $0.50( \pm 0.30)$ & $0.70( \pm 0.25)$ \\
$\alpha=90(\mathrm{deg})$ & $0.70( \pm 0.30)$ & $1.10( \pm 0.35)$ & $1.50( \pm 0.40)$ \\
$\alpha=135(\mathrm{deg})$ & $1.30( \pm 0.30)$ & $1.80( \pm 0.40)$ & $2.15( \pm 0.45)$ \\
$\alpha=180(\mathrm{deg})$ & $1.60( \pm 0.25)$ & $2.00( \pm 0.40)$ & $2.30( \pm 0.30)$ \\
\hline
\end{tabular}

Personal Space Distances. Average distance values between Tibi and volunteers, given in meters.

to skip the test at any time. Once the experiment was finished, participants answered the questionnaire.

Human-Assisted Face Recognition. The face recognition system used in our experiments is based on the classifier proposed in [20]. This classifier, dubbed Online Random Ferns, interactively computes a discriminative detector that allows to recognize objects and human faces in real-time. Although this classifier was shown to improve the recognition performance with higher rates of human assistance, the work described in [20] did not explicitly evaluate the influence of the human intervention over the human-robot interaction.

In this paper, we extended [20] with an empirical and quantitative evaluation of the human assistance from the perspective of HRI. The evaluation was carried out based on the interactions between the Tibi robot and several persons in a variety of environmental conditions. Fig. 8 shows a few sample images of such experiments.

More specifically, we evaluated the human-robot interaction for online face recognition in terms of the degree of human intervention. To this end, we followed the criterion used in [20], where a confidence interval $\theta$ was set to decide when the human intervention was required, this used as a human assistance interval. Yet, while in [20] this threshold was set to a fixed value, here we have evaluated the face recognition module using different values of $\theta$, and thus, different amounts of human intervention.

\section{Results}

We next present the results for each of the experiments. Note that the three experiments are interconnected, i.e., the results of the "People's personal space" are fed into the "Robot's behaviors" experiments, which in turn are used to carry out the "Human's Assistance Face Recognition" tests.

\section{A. People's personal space}

As mentioned above, the first part of the experiments aimed to determine the personal space desired by people when they interact with the robot. To that end, we conducted a series of tests in which the robot moved towards a person at different speeds $(0.3 \mathrm{~m} / \mathrm{s}, 0.6 \mathrm{~m} / \mathrm{s}$ and $0.9 \mathrm{~m} / \mathrm{s})$ and at different angles $\left(0^{\circ}, 45^{\circ}, 90^{\circ}, 135^{\circ}\right.$ and $\left.180^{\circ}\right)$.

Table IV shows the distances of the person's vital space, for each speed and angle value. It can be observed, that if the speed increases the distances become larger, and, therefore, the size of the personal space increases. People's personal space also depends on the approaching angle, requiring smaller distances when the robot faces directly the person.
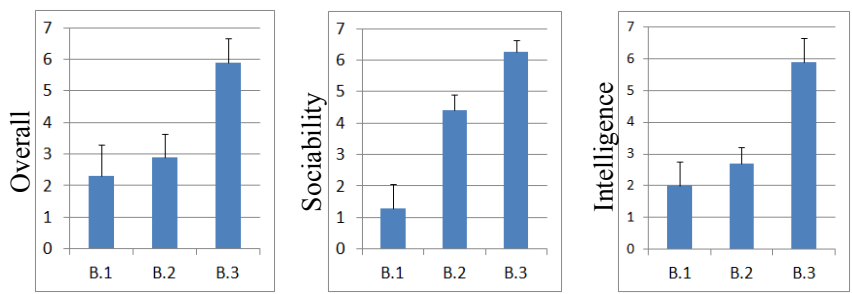

Fig. 5. HRI Results. Degree of acceptance of the three robot's behaviors. Left: Global evaluation of the strategies. Center: Robot's sociability. Right: Robot's intelligence perceived by the humans.

The graphs show the magnitude of the personal space as a function of the robot velocity and approaching angle.

These tests were performed both indoor and outdoor, and no special requirements were made about the properties of the scenarios. During the approaching phase, there was just a person within the robot's neighborhood. The angle at which the volunteers moved away from the robot changed randomly in each experiment. And, when the robot was behind the person, the robot did use its verbal cues to attract attention.

The results of the personal space were then incorporated into the robot's behavior when performing the interaction process of the subsequent experiments.

\section{B. Robot's behavior}

As mentioned before, each participant filled out a questionnaire. The measurement was a simple rating on a Likert scale between 1 to 7 . In this section, we provide the results of comparing the following three robot behaviors: $(B 1)$ the robot only uses verbal communication; $(B 2)$ the robot uses verbal communication and gestures; and (B3) the robot uses verbal, nonverbal communication and may approach the person.

For the global evaluation score plotted in Fig. 5-Left, repeated ANOVA measures were conducted. A significant main effect was found, $F(2,27)=38.23, p<0.001, \eta^{2}=$ 0.27. Multiple comparisons with the Bonferroni method revealed that the score for $B 3$ is significantly higher than both behaviors $B 1(p<0.001)$ and $B 2(p<0.001)$. No significant difference was found between $B 1$ and $B 2(p=0.224)$.

To analyze the source of the difference, additional scores were examined. For the robot's sociability (Fig. 5-Center) a repeated-measures analysis of variance revealed a significant effect, $F(2,27)=139.30, p<0.001$, partial $\eta^{2}=0.1$. Pairwise comparison with Bonferroni showed a remarkable difference between the three strategies as well. $B 1$ vs. $B 2$ : $p<0.01$; B1 vs. B3: $p<0.001$; B2 vs. B3: $p<0.001$.

Finally, for the robot's intelligence (Fig 5-Right), a repeated-measures analysis of variance revealed a significant main effect, $F(2,27)=27.15 p<0.001$, partial $\eta^{2}=0.33$. Pairwise comparison with Bonferroni revealed that the score for $B 3$ is significantly higher than both $B 1(p<0.001)$ and $B 2(p=0.0015)$ strategies. No significant difference was found between $B 1$ and $B 2(p=0.33)$.

As a summary, after analyzing the three different behaviors, we may conclude that when the robot uses verbal and nonverbal communication, and is able to approach the person, it has the largest acceptance. People perceived the robot to be more intelligent, as it could detect and approach them, and, they felt that it had more social skills. Fig. 6 


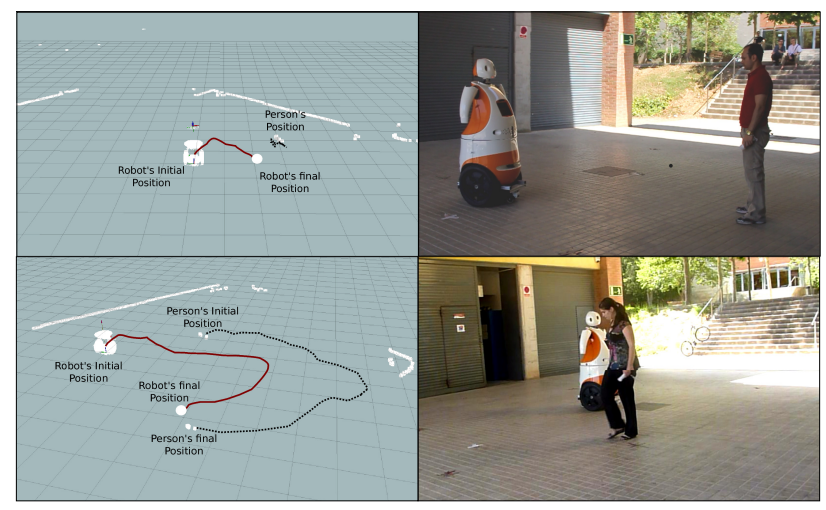

Fig. 6. Tibi Starts an Interaction. The robot approaches two people and begins an interaction with them. Top: The person is waiting for Tibi. Bottom: The person is moving and Tibi follows her and invites her.

shows a sample path followed by a robot when approaching a person. In the upper images, the person is waiting for Tibi, and in the lower images the robot is inviting the person, who is walking, to start the interaction.

\section{Human-Assisted Face Recognition}

The online human-assisted face recognition system was evaluated in terms of the degree of human intervention and its effects over the interaction between the Tibi and human users. In particular, we have focused on the duration of the established interaction and users' comfortability.

Fig. 7 shows the impact of the human assistance over the human-robot interaction. The left figure corresponds to the percentage of human intervention for different assistance intervals $\theta$. We see that the assistance percentage increases as the interval gets larger. This means that people are more active in the interaction and help the robot to a greater extent during the learning stage. However, it is at the expense of a greater effort from human users. This fact results in shorter interactions since people get tired rapidly and lose interest in helping to the robot to compute a robust face recognizer. Fig. 7-Right shows this behavior by means of the average interaction and assistance times. As the degree of human assistance gets larger (i.e., interval size), the interaction time between the robot and humans gets shorter. It is also worth to observe that the interaction time with a pretty small percentage of human intervention is relatively short. This is because, as the human participation is minor (i.e., human users seldom help the robot), people also lose interest in the cooperative and interactive task of face learning and recognition. We have found that a satisfactory compromise between human's effort and interaction time is achieved for an assistance interval of $\theta=[0.4,0.6]$.

Finally, the face recognition rates obtained by the proposed method in terms of human assistance are given in Table V. The recognition rates are consistently very high. This experiment confirmed the recognition results reported in [20], where the human assistance improves the face classifier during the human-robot interaction. Furthermore, once the face classifier has been learned, it runs in real-time in the Tibi robot. Fig. 8 shows example images with the output of the face recognizer.
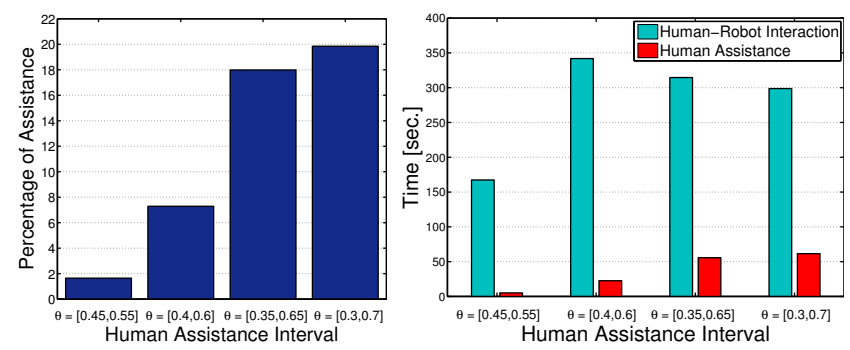

Fig. 7. Human Assistance. Left: Percentage of human assistance in the face recognition system according to varying assistance intervals. Right: Average times spent for human-robot interaction and human-assistance.

\section{DISCUSSION}

The findings presented in the previous section reinforce the idea that robot's ability to initiate the interaction is an important skill to let it naturally interact with people. Overall, people were surprised to find a robot in a public space, and they were astonished because the robot catched their attention. Moreover, they enjoyed helping the robot to recognize their faces and were surprised to see how the robot progressively improved its visual skills with their assistance.

We have found concluding results for all the three experiments we have performed. First, we computed the personal area required by the persons when a robot was approaching to them. The obtained results were consistent in all volunteers, and were then integrated within the robot behavior model.

Secondly, we found that people feel more natural the interaction with the robot when it is performed through gestures, speech and motion. Detailed analysis showed that robot's capabilities improved the perception of the robot's intelligence and sociability. Furthermore, the amount of speech and comments of the robot seem to be appropriate for this type of scenario. Moreover, people felt comfortable using the Wii remote control to communicate with the robot.

Thirdly, we have proven that the human assistance leads to compute robust classifiers to recognize human faces. The learning and detection are performed efficiently and with minimal human effort. The results show that using a social robot, the interest of people increases and they help Tibi to improve its visual skills.

We noticed that very few participants were capable of enumerating disadvantages of the robot, but most of them provided many suggestions when asked about improvements for Tibi. People would like to communicate with the robot via voice commands, since communication would be more comfortable. Furthermore, people suggest that it would be interesting if they could teach other objects in the scene pointing them at the robot's screen. Both these remarks will be part of our future work.

Finally, it is worth to point that the parameters obtained in the first set of experiment to describe human's personal space deal with European people and our own robot. Therefore, when it is adapted, one would need to consider adapting parameters. Moreover, the proposed model of interaction was tested in a specific scenario, hence its generalization is somehow limited. It is possible that the context affects the preference of a specific robot behavior. For example, in a business environment, a mobile robot approaching people 
TABLE V

\begin{tabular}{|c|c|c|c|c|}
\hline \multicolumn{5}{|c|}{ Face Recognition Results } \\
\hline & \multicolumn{4}{|c|}{ Human Assistance Interval $\theta$} \\
\cline { 2 - 5 }$[0.55,0.65]$ & {$[0.4,0.6]$} & {$[0.35,0.65]$} & {$[0.3,0.7]$} \\
\hline $\begin{array}{c}\text { Recognition } \\
\text { Rate }\end{array}$ & $99.50 \%$ & $98.74 \%$ & $97.74 \%$ & $98.41 \%$ \\
\hline $\begin{array}{c}\text { Percentage of } \\
\text { Assistance }\end{array}$ & $1.65 \%$ & $7.28 \%$ & $17.98 \%$ & $19.84 \%$ \\
\hline $\begin{array}{c}\text { Interaction } \\
\text { Time [sec.] }\end{array}$ & 167.4 & 341.8 & 314.5 & 298.7 \\
\hline
\end{tabular}

Human-Assisted Face Recognition Results. The face recognition system was evaluated in terms of the degree of human intervention $\theta$.

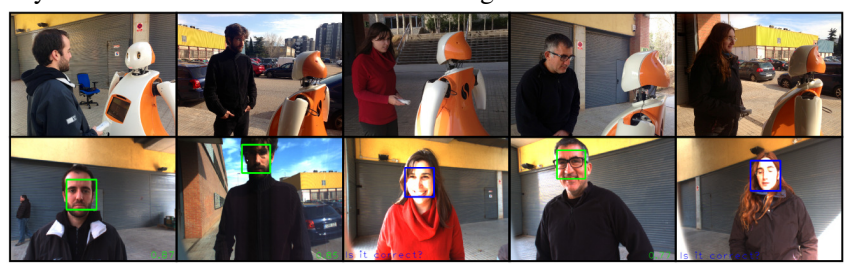

Fig. 8. Face Recognition. Top: People interacting with the Tibi robot. Bottom: Tibi's field of vision. Face detections are represented by green boxes, whereas blue ones indicate that the system requires the human assistance.

can be annoying, as it could disturb people. We believe that the University Campus is rather neutral, so it could reflect interaction in many daily use scenarios.

\section{CONCLUSIONS}

We have presented an autonomous mobile robot seeking interaction for human-assisted learning. The contributions of the paper are two-fold. First, we have studied different robot behaviors to initiate interaction with humans. The robot was able to autonomously approach a person and create an engagement with him/her.

Second, once the engagement was created, people could assist the social robot to improve its face recognition system. During the interaction, the robot is continuously learning and refining its face models in order to get a robust and discriminative classifier with which to detect faces in difficult scenarios. The human assistance is essential in those cases where the robot is not confident about its predictions.

Both contributions have been extensively and rigorously tested in a real environment. The findings suggest that allowing the robot to take initiative when communicating with people, the number of human-to-robot interactions increases. This, in its turn, leads the humans to assist robot to improve its visual skills, and perform more reliable interactions.

\section{REFERENCES}

[1] K.O. Arras, O.M. Mozos, and W. Burgard. Using boosted features for the detection of people in $2 \mathrm{~d}$ range data. In ICRA, 2007.

[2] H.H Clark. Using language. Cambridge University Press Cambridge, 1996.

[3] B. Cooper, P. Brna, and A. Martins. Effective affective in intelligent systems-building on evidence of empathy in teaching and learning. Affective interactions, 2000.

[4] K. Dautenhahn, M. Walters, S. Woods, K.L. Koay, C.L. Nehaniv, A. Sisbot, R. Alami, and T. T. Siméon. How may i serve you?: a robot companion approaching a seated person in a helping context. In SIGCHI/SIGART, 2006.
[5] A. Garrell and A. Sanfeliu. Model validation: robot behavior in people guidance mission using dtm model and estimation of human motion behavior. In IROS, 2010.

[6] A. Garrell and A. Sanfeliu. Cooperative social robots to accompany groups of people. In IJRR, 2012.

[7] A. Garrell, A. Sanfeliu, and F. Moreno-Noguer. Discrete time motion model for guiding people in urban areas using multiple robots. In IROS, 2009.

[8] E. Goffman. Behavior in public places: Notes on the social organization of gatherings. 1966.

[9] E.T. Hall. The hidden dimension, man's use of space in public and private. Great Britain, London: The Bodley Head Ltd, 1966.

[10] R. Kirby. Social robot navigation. PhD thesis, Carnegie Mellon University, The Robotics Institute, 2010.

[11] M.P. Michalowski, S. Sabanovic, and R. Simmons. A spatial model of engagement for a social robot. In IEEE Int. Workshop on Advanced Motion Control, 2006.

[12] Y. Morales, S. Satake, R. Huq, D. Glass, T. Kanda, and N. Hagita. How do people walk side-by-side?: using a computational model of human behavior for a social robot. In HRI, 2012.

[13] F. Moreno-Noguer, A. Sanfeliu, and D. Samaras. Integration of deformable contours and a multiple hypotheses fisher color model for robust tracking in varying illuminant environments. In Image and Vision Computing, 2007.

[14] B. Mutlu, T. Shiwa, T. Kanda, H. Ishiguro, and N. Hagita. Footing in human-robot conversations: how robots might shape participant roles using gaze cues. In HRI, 2009.

[15] A. Sanfeliu and J. Andrade-Cetto. Ubiquitous networking robotics in urban settings. In Proc. of the IEEE/RSJ IROS Workshop on Network Robot Systems, 2006.

[16] S. Satake, T. Kanda, D.F. Glas, M. Imai, H. Ishiguro, and N. Hagita. How to approach humans?: strategies for social robots to initiate interaction. In HRI, 2009.

[17] C. Shi, M. Shimada, T. Kanda, H. Ishiguro, and N. Hagita. Spatial formation model for initiating conversation. In RSS, 2011.

[18] C. Trevai, Y. Fukazawa, J. Ota, H. Yuasa, T. Arai, and H. Asama. Cooperative exploration of mobile robots using reaction-diffusion equation on a graph. In ICRA, 2003.

[19] M. Villamizar, J. Andrade-Cetto, A. Sanfeliu, and F. Moreno-Noguer. Bootstrapping boosted random ferns for discriminative and efficient object classification. Pattern Recognition, 2012.

[20] M. Villamizar, A. Garrell, A. Sanfeliu, and F. MorenoNoguer. Online human-assisted learning using random ferns. In ICPR, 2012.

[21] M.L. Walters, D.S. Syrdal, K.L. Koay, K. Dautenhahn, and R. te Boekhorst. Human approach distances to a mechanical-looking robot with different robot voice styles. In RO-MAN, 2008. 